\title{
An Integrative Psychophysiological Study of Cognitive Function in Active Elderly: Rationale, Methods and Initial Results
}

\author{
Shuzhen Zhang ${ }^{*}$, Yukihiro Yada ${ }^{2}$, Akira Tsuda ${ }^{3}$ \\ ${ }^{1}$ Graduate School of Psychology, Kurume University, Kurume, Japan \\ ${ }^{2}$ School of Integrative and Global Majors, University of Tsukuba, Tsukuba, Japan \\ ${ }^{3}$ Department of Psychology, Kurume, Japan \\ Email: ding791017@sohu.com
}

Received 11 August 2015; accepted 12 September 2015; published 18 September 2015

\begin{abstract}
One of the critical issues is that Japan is well known for the world's highest proportion of elderly people, a super-aged society, and is currently confronting with preventing physical and psychological disability in elderly. In order to reduce the potential risk, we wish the guidance will be suggested to help active elderly around 65 years old have healthy daily life with high quality.
\end{abstract}

\section{Keywords}

Active Elderly, Cognitive Function, Perceived Health, QOL, Integrative Psychophysiological Study

\section{Introduction}

Recently, Japan is facing the problems with the low birthrate and aging population. In 2014, people aged 65 years old or older accounted for $25.1 \%$ of Japanese entire population. This number will increase steadily, and the elderly are expected to account for $33.4 \%$ of the country's total population [1]. As a result, the medical expenses for the elderly keep on increasing year by year. As the same time, we are also facing the problems such as the closedown of the medical institution and the collapse of the public pension system.

Government shows the guidance for health promotion and health maintenance. Lots of measures have been carried out to promote the health food, support the elderly facilities for health maintenance, develop the health food with private companies such as health food makers, and develop health support programs in medical facilities.

\section{Aim}

The aim of this study is not only to reduce the health risk which will be hardly with aging in the future, but also to propose the general support program of the living evaluation which is necessary to improve the subjective health of the young-old persons according to the analysis of mental stress, activities of daily living and physical function (central function-autonomic nervous function-Periphery-somatic sensation) in the healthy young-old (active elderly). Furthermore, we wish that the health evaluation support programs with global viewpoint will be introduced to countries of East Asia such as China, Indonesia. 


\section{Significance}

When looking at the living conditions or physical functions of the elderly persons, there are differences in not only age, sex, but also in the living environment, family constitution, the relation with the communities, lifestyle and the illness [2]. Especially, in middle-old aged persons, there are large differences in mental and physical state. Therefore, it's necessary to provide mental and physical care for everyone. However, now, it's hard to be realized. Though, Japan has tried to cope with the aged society arrived by lots of measures, there are few concrete preparations for the arrival of the super-aged society. So, it seems that the early healthy supports for the young-old persons (born in 1955-1957) become more and more important in the future.

The young-old persons called activity elderly by media are highly concerned with lots of hobbies or trips. There are many people who are active in self-management of health or enlightenment [3] [4]. The supports for both body and mind of activity elderly will improve the subjective health of each person. It also will be a powerful measure to deal with the super-aged society not far.

By the way, subjective health of elderly persons is related with life satisfaction or happiness [5]. It's useful as a substitute for medical health index [6]. In other words, it's an important problem to raise the subjective heath of elderly persons in the elderly persons' health promotion.

On the other hand, it's well known that the decline in cognitive function or physical/mental function will arise with aging [7]. As a drop of the physical ability, the reduction in strength of knees or elbow's bend is due to the drop of the muscle mass of leg's skeletal muscles. Furthermore, general functions and sensuous coordination will fall caused by the drop of mental or cognitive function, such as thought, judgment, memory, incentive, emotion and so on. As a result, with walking motion (walking speed, walking ratio; decrease in step size, expansion of walking interval) disturbed, it's may cause a fall by the drop of the walking attention [8] [9]. So, if we want to make clear the relationship between physical state and mental state of elderly persons, we must to analyze the data by using physiological and psychological indexes.

\section{The Hypothesis of Study}

From the researches of cognitive function, it's suggested that the drop of the walking speed and grip is an index to judge the decline in cognitive function. So, it's can be thought about that the relations of mental and cognitive function are so important to keep up the physical functions. It's necessary for us to analyze the relationship between physical abilities and the drop of mental or cognitive function from physiological and psychological viewpoint.

\section{Study Project}

\subsection{Subject}

Healthy active seniors living in Fukuoka or nearby the joint research institutes.

\subsection{Summary of Evaluation}

Evaluate mental and physical state or function by physiologic and psychological index generally.

\section{Evaluation Indexes}

- Physiological evaluation indexes

1) Autonomic nerve activity: analyze the heartbeat change with electrocardiogram; analyze the blood pressure variation with blood pressure measurement; analyze the miosis rate by the pupil-to-light meter.

2) Cerebral activity: analyze the brain function based on the measurement of the brain blood flow with far-red light; analyze the cognitive function with the brain executive function meter; analyze the fatigue of the cerebrum with flicker meter.

3) Walking motion: analyze the continuous walking with the sheet type walk analyzer.

4) Centroid oscillation: analyze the general date though standing, sitting and continuous action with sitting stabilometer.

5) Immune response: analyze the anti-stress hormone of saliva (cortisol, HMEC, chromogranin A, etc.)

6) General function: analyze the sleeping station with sheet type sleep determining apparatus.

- Psychological evaluation indexes 
1) Questionnaire: analyze the subjective well-being (positive feeling) and mental fatigue (negative feeling) with SUBI (The Subjective Well-being Inventory); analyze the degree of mental health and neurological symptom with GHQ (World health organization, The General Health Questionnaire); analyze subjective stress awareness with SCL30 (stress check list 30); analyze the Independence degree of living activities with ADL (Activities of Daily Living); analyze the state and transformation of quality of living with SF36 (health-related QOL scale).

2) Test: analyze the attention of elderly by TMT-A (Trail Making Test part A); measure brain age and brain stress by Brain Age Meter-ATMT (measure reaction time by touching continuous numbers).

\subsection{Study Schedule}

- 2015.4-2016.3

1) Before the examination in elderly people, this study target at students and teachers of Kurume University with agreement. To improve the technical skills with the physiological and psychological evaluation index of the above and to verify the items timely.

2) Make measurements or examinations for elderly people at elderly facilities or home about the items every 3-4 months (Four times a year and two years continuously).

- 2016.4-2017.3

1) Analyze the relationship between psychological evaluation indexes such as the subjective health, stress awareness of the subjects, and physiological evaluation indexes such as walking motion or cognitive function. (One year later)

1) Carry out various kinds of interventions (bathing, sleep, hyperthermia, light incontinence pad, walk instruction, fragrance) in elderly people with agreement and verify the effect.

- 2017.4-2018.3

1) Analyze and evaluate the effectiveness basic the date of psychological and physiological evaluation indexes with using one-way analysis of variance, multivariate analysis and logistic analysis.

2) Summarize and consider the result of study from the second year, and post it to the associated society.

\subsection{Initial Results}

- We had a pre-examination in April 2015. The following instruments were used for data collection. Apparatus: Brain Age Mete-ATMT; Questionnaires of reacted-QOL: Instrumental ADL; SF-8 ${ }^{\mathrm{TM}}$; Mini-Mental State Examination (MMSE); GDS5 (Geriatric Depression Scale 5); Forgetfulness questionnaire; Sleep state (hours of sleep, times of nocturnal awakening, subjective sleep state).

The subjects in Table 1 consisted of 14 male and 34 female elderly with an average age of 80.9 (range: 65 -

Table 1. Characteristics of subjects.

\begin{tabular}{|c|c|c|}
\hline \multicolumn{3}{|c|}{ Characteristics of Subjects $(\mathrm{N}=48 \mathrm{M}=14 \mathrm{~F}=34)$} \\
\hline & Average of Subjects & SD \\
\hline Age & 80.9 & 6.9 \\
\hline Brain Age & 74.7 & 7.9 \\
\hline Instrumental ADL (0 - 5) & 4.81 & 0.53 \\
\hline Intellectual ADL (0 - 4) & 3.77 & 0.47 \\
\hline Social ADL (0 - 4) & 3.19 & 1.02 \\
\hline IADL Total Score (0 - 13) & 11.77 & 1.49 \\
\hline Depressive Symptom (0 - 5) & 0.83 & 1.1 \\
\hline Forgetfulness (0 - 6) & 0.71 & 0.87 \\
\hline SF-8 Physiological Summary Score & 48.56 & 7.19 \\
\hline SF-8 Psychological Summary Score & 52.04 & 6.55 \\
\hline Hours of Sleep & 8.23 & 1.66 \\
\hline Times of Nocturnal Wakening & 1.29 & 1.15 \\
\hline Subjective Sleep State (4 - 20) & 15.6 & 2.89 \\
\hline MMSE Total Score $(0$ - 30) & 27.34 & 2.82 \\
\hline
\end{tabular}


93). The average brain age by using ATMT was 74.7. MMSE score was good (cut-off point: 23/24). The IADL score and SF-8 summary score were higher than national standard or the average of precedent study. The score of depressive symptom or forgetfulness was fine.

\section{Conclusion}

In the near future, the super-aged society will come in global volume. By the study, we hope to suggest the evaluation technologies or support programs which will be widely utilized as the elderly evaluation system with global view.

\section{References}

[1] http://www8.cao.go.jp/kourei/whitepaper/w-2015/zenbun/27pdf_index.html

[2] Miyabara, H. and Oda, T. (2007) Relationships among Lifestyle, Motor Ability, Functional Capacity and Social Attributes in Old Age. Rigakuryoho Kagaku, 22, 397-402. http://dx.doi.org/10.1589/rika.22.397

[3] Endo, H. and Matsuyuki, Y. (2005) Life Courses in Aged Society and Unpaid Works by Active Seniors. Studies in Regional Science, 35, 439-450.

[4] Endo, H. (2011) A Case-Study on Social Business in Japan. Collection of the studies of Kaetsu University, 53, 45-62. http://ci.nii.ac.jp/naid/110008427681/

[5] Igarashi, H. and Iijima, S. (2006) Effects of Life-Style Factors and Health Related Factors on Subjective Health in Male Workers. Yamanashi Nursing Journal, 4, 19-24. http://opac.lib.yamanashi.ac.jp/opac/repository/1/21000/KJ00004493560.pdf

[6] Mitoku, K., Takahashi, T. and Hosi, T. (2006) Reviewing the Studies of the Relation between Feeling of Health and Mortality. Kawasaki Medical Welfare Journal, 16, 1-10. http://www.kawasaki-m.ac.jp/soc/mw/journal/jp/2006-j16-1/02_mitoku.pdf

[7] Baddely, A. (2003) Working Memory: Looking Back and Looking Forward. Nature Reviews Neuroscience, 4, 829-839. http://dx.doi.org/10.1038/nrn1201

[8] Kuno, K.J., Soma, R., Masuda, K., Adachi, K., Nishijima, T., Ishizu, M. and Okada, M. (2000) Relationship between Reduction of Hip Joint and Thigh Muscle and Walking Ability in Elderly People. Japanese Journal of Physical Fitness and Sports Medicine, 5, 589-596. https://www.jstage.jst.go.jp/article/jspfsm1949/49/5/49 5 589/ article

[9] Murata, S., Otao, H., Murata, J., Horie, J., Miyazaki, J. and Mizota, K. (2009) A Prospective Study of Relationships between Fall-Related Accidents in the Elderly Living in the Community and their Physical, Psychological, and Cognitive Functions. Rigakuryoho Kagaku, 24, 807-812. http://dx.doi.org/10.1589/rika.24.807 